\title{
A Kleene Analysis of Mobile Ambients
}

\author{
Flemming Nielson ${ }^{1}$, Hanne Riis Nielson ${ }^{1}$, and Mooly Sagiv ${ }^{2}$ \\ 1 Aarhus University \\ 2 Tel Aviv University
}

\begin{abstract}
We show how a program analysis technique originally developed for $C$-like pointer structures can be adapted to analyse the hierarchical structure of processes in the ambient calculus. The technique is based on modeling the semantics of the language in a two-valued logic; by reinterpreting the logical formulae in Kleene's three-valued logic we obtain an analysis allowing us to reason about may as well as must properties. The correctness of the approach follows from a general Embedding Theorem for Kleene's logic; furthermore embeddings allow us to reduce the size of structures so as to control the time and space complexity of the analysis.
\end{abstract}

\section{Introduction}

Mobile ambients. The ambient calculus is a prototype web-language that allows processes (in the form of mobile ambients) to move inside a hierarchy of administrative domains (also in the form of mobile ambients); since the processes may continue to execute during their movement this notion of mobility extends that found in Java where only passive code in the form of applets may be moved. Mobile ambients were introduced in [1] and have been studied in 20349131617. The calculus is patterned after the $\pi$-calculus but focuses on named ambients and their movement rather than on channel-based communication; indeed, already the communication-free fragment of the calculus is very powerful (and in particular Turing complete); we review it in Section 2

Since processes may evolve when moving around it is hard to predict which ambients may turn up inside what other ambients. In this paper we present an analysis that allows us to validate whether all executions satisfy properties like:

- Is there always exactly one copy of the ambient $p$ ?

- Is $p$ always inside at most one of the ambients $r_{1}, r_{2}$ and $r_{3}$ ?

Kleene's three-valued logic. In [18] Kleene's three-valued logic is used to obtain safe approximations to the shape of dynamically evolving C-like pointer structures. From a programming language point of view, the setting of the present paper is vastly different. In contrast to traditional imperative languages the ambient calculus has no separation of program and data, furthermore non-determinism and concurrency are crucial ingredients and hence the notion of program point is demoted. The central operations on C-like pointers are assignments, which are executed one at a time and with a local effect on the heap; 
this is in contrast to the reductions of the ambient calculus which may happen in a number of not a priori known contexts - thus the overall effect is hard to predict. We present an overview of Kleene's three-valued logic in Section 3 .

Predicate logic as a meta-language. Our overall approach is a meta-language approach as known for example from the denotational approach to program analysis [14]. However, here we are based on a predicate logic with equality and appropriate non-logical predicates as well as operations for their transitive closure; the choice of non-logical predicates is determined by the programming language or calculus at hand. To deal with equality we rely on the presence of a special unary summary predicate indicating whether or not an individual represents one or more entities. The important point is that the logic must be powerful enough to express both

- the properties of the configurations that we are interested in, and

- a postcondition semantics for transitions between configurations.

From a process algebra point of view, our representation of ambients (presented in Section 4) is rather low-level as it operates over structures containing a universe of explicit individuals; the non-logical predicates are then used to encode mobile ambients within these structures. The benefit of using sets of individuals, over the more standard formulation using multisets of subambients, is that it directly allows us to use the logical formulae.

Static analysis. The aim of the analysis is to identify certain invariant properties that hold for all executions of the system; from the process algebra point of view, the invariants are akin to types and they represent the sets of ambients that can arise. Since the set of ambient structures may be infinite, the analysis needs to perform an abstraction to remain tractable.

For a moment let us assume that we continue interpreting the specification in a two-valued logic. From the classical program analysis point of view, the maxim that "program analysis always errs on the safe side" amounts to saying that the truth values false and true of the concrete world are being replaced by 0 (for false or cannot) and a value $1 / 2$ (for possibly true or may) in the abstract world. As an example, if a reachability analysis says that a given value (or ambient) cannot reach a given point (or ambient) then indeed it cannot, but if the reachability analysis says that it might then it may be a "false positive" due to the imprecision of the analysis.

The Embedding Theorem. The power of our approach comes from the ability to reinterpret the transition formulae over Kleene's three-valued logic. Here we have the truth values 0 (for false or cannot), 1 (for true or must) and $1 / 2$ (for possibly true or may) [18]. The benefit of this is that we get a distinction between may and must properties for free! Returning to the reachability example we are now able to express certain cases where a value (or ambient) definitely reaches a given point. 
It is straightforward to reinterpret the specification of the semantics over structures that allow all three truth values (see Section 5). The correctness of the approximate analysis with respect to the semantics follows using a general Embedding Theorem that shows that the interpretation in Kleene's three-valued logic is conservative over the ordinary two-valued interpretation. Termination is guaranteed thanks to our techniques for restricting attention to a bounded universe of individuals and for combining certain structures into one (thereby possibly introducing more 1/2's); these techniques generally work by allowing us to compress structures so that fewer individuals (perhaps only boundedly many) are needed and thereby allowing us to control the time and space complexity of our analysis.

\section{The Ambient Calculus}

Syntax and informal semantics. We shall study the communication-free subset of the ambient calculus 12 and for simplicity of presentation we shall follow [2] in dispensing with local names. Given a supply of ambient names $n \in N$ we define the syntax of processes $P \in$ Proc and capabilities $M \in$ Cap:

$$
\begin{aligned}
& P::=0\left|P \llbracket P^{\prime}\right| ! P|n[P]| M . P \\
& M::=\text { in } n \mid \text { out } n \mid \text { open } n
\end{aligned}
$$

The first constructs are well-known from the $\pi$-calculus. The process 0 is the inactive process; as usual we shall omit trailing 0 's. We write $P \llbracket P^{\prime}$ for the parallel composition of the two processes $P$ and $P^{\prime}$ and we write $! P$ for the replicated process that can evolve into any number of parallel occurrences of $P$.

The remaining constructs are specific to the ambient calculus. The construct $n[P]$ encapsulates the process $P$ in the ambient $n$. In the basic ambient calculus a process can perform three operations: it can move into a sibling ambient using the in $n$ capability, it can move out of the parent ambient using the out $n$ capability or it can dissolve a sibling ambient using the open $n$ capability. These operations are illustrated pictorially in Fig. 1 where we draw the processes as trees: the nodes of the trees are labelled with names and capabilities and the subtrees represent parallel processes "inside" the parent. The figure expresses that when a process matches the upper part of one of the rules then it can be replaced by a process of the form specified by the lower part. The reduction can take place in a subprocess occurring deeply inside several ambients; however, capabilities always have to be executed sequentially.

Example 1. Throughout the paper we shall consider the following example:

$$
\begin{aligned}
p\left[\text { in } r_{1} \| \text { ! open } r\right] & \| r_{1}\left[! r\left[\text { in } p \text {. out } r_{1} \text {. in } r_{2}\right]\right] \\
& r_{2}\left[! r\left[\text { in } p \text {. out } r_{2} . \text { in } r_{3}\right] \| ! r\left[\text { in } p \text {. out } r_{2} \text {. in } r_{1}\right]\right]
\end{aligned}
$$

It illustrates how a package $p$ is first passed to the site $r_{1}$, then to $r_{2}$ from which it is either passed to $r_{3}$ or back to $r_{1}$. 


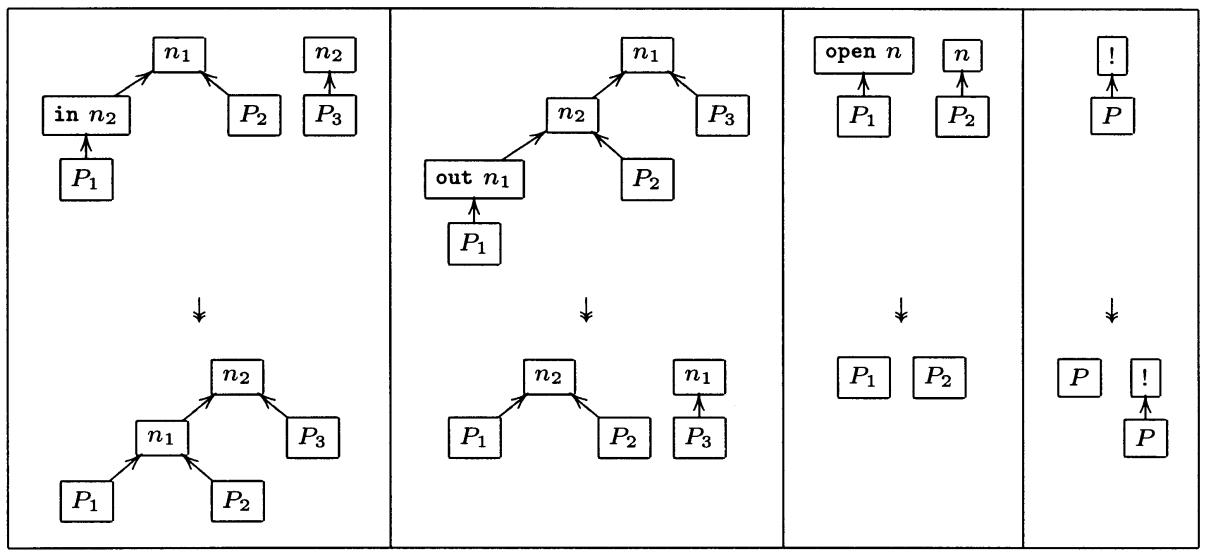

Fig. 1. Semantics of capabilities and replication.

Formal semantics. Formally the semantics is specified by a structural congruence relation $P \equiv Q$ allowing us to rearrange the appearance of processes (e.g. corresponding to reordering the order of the descendants of a node in a tree) and a reduction relation $P \rightarrow Q$ modelling the actual computation; the only deviation from [1] is that the semantics of replication is part of the transition relation rather than the congruence relation (see Fig. 1).

\section{A Primer on Three-valued Logic}

Syntax. It will be convenient to use a slight generalization of the logic used in [18. Let $\operatorname{Pr}[k]$ denote the set of predicate symbols of arity $k$ and let $\operatorname{Pr}=$ $\bigcup_{k} \operatorname{Pr}[k]$ be their finite union. We shall write $=$ for the equality predicate and furthermore we shall assume that $\operatorname{Pr}[1]$ contains a special predicate $s m$. Here $s m$ stands for "summary-predicate" and we shall later interpret it as meaning that its argument might represent multiple individuals. Without loss of generality we exclude constant and function symbols from our logic; instead we encode constant symbols as unary predicates and $n$-ary functions as $n+1$-ary predicates.

We write formulae over $\operatorname{Pr}$ using the logical connectives $\vee, \neg$ and the quantifier $\exists$; the formal syntax is:

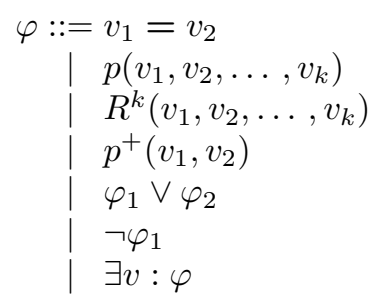

equality on individuals predicate value, $p \in \operatorname{Pr}[k]$ application of second order free variable transitive closure of a relation $p, p \in \operatorname{Pr}[2]$

disjunction negation

(first order) existential quantification

Capital letters of the form $R^{k}$ are used for (second-order) relations of arity $k$. We also use several shorthands: $\forall v: \varphi$ stands for $\neg \exists v: \neg \varphi$ and $\varphi_{1} \wedge \varphi_{2}$ stands for 
Table 1. Kleene's three-valued interpretation of the propositional operators.

\begin{tabular}{|c|c|c|c|c|c|c|c|}
\hline$\wedge$ & $\mid \begin{array}{ll}0 & 1\end{array}$ & $1 / 2$ & V & 0 & $11 / 2$ & & $\neg$ \\
\hline 0 & 0 & $\begin{array}{ll}0 & 0\end{array}$ & 0 & 0 & $111 / 2$ & 0 & 1 \\
\hline 1 & $\begin{array}{ll}0 & 1\end{array}$ & $1 \quad 1 / 2$ & 1 & & 11 & 1 & 0 \\
\hline $1 / 2$ & $\left|\begin{array}{ll}0 & 1\end{array}\right|$ & $/ 21 / 2$ & $1 / 2$ & $1 / 2$ & $11 / 2$ & $1 / 2$ & $1 / 2$ \\
\hline
\end{tabular}

$\neg\left(\neg \varphi_{1} \vee \neg \varphi_{2}\right)$. The above shorthands are useful since three-valued logic formulae obey De-Morgan laws. Also $\varphi_{1} \Longrightarrow \varphi_{2}$ stands for $\left(\neg \varphi_{1} \vee \varphi_{2}\right)$ and $v_{1} \neq v_{2}$ stands for $\neg\left(v_{1}=v_{2}\right)$. Finally, we assume the standard associativity and precedence rules.

Semantics. A two-valued interpretation of the language of formulae over $\operatorname{Pr}$ is a structure $T=\langle U, \iota\rangle_{2}$, where $U$ is a set of individuals and $\iota$ maps each predicate symbol $p$ of arity $k$ to a truth-valued function:

$$
\iota: \operatorname{Pr}[k] \rightarrow U^{k} \rightarrow\{0,1\} .
$$

A three-valued interpretation is then a structure $T=\langle U, \iota\rangle_{3}$, where now $\iota$ maps each predicate symbol $p$ of arity $k$ to a truth-valued function:

$$
\iota: \operatorname{Pr}[k] \rightarrow U^{k} \rightarrow\{0,1,1 / 2\} .
$$

We use Kleene's three-valued semantics which operates with the three values: 0, 1 and $1 / 2$. The values 0 and 1 are called definite values and $1 / 2$ is an indefinite value. The informal semantics of this logic is given in Table 1 where $1 / 2$ represents situations were the result may be either true or false. Alternatively, think of 1 as representing $\{$ true $\}, 0$ as representing $\{$ false $\}$, and $1 / 2$ as representing $\{$ false, true $\}$. In the propositional case, our presentation of three-valued logics here follows [7, Chapter 8]. We shall omit the subscripts ${ }_{2}$ and ${ }_{3}$ when it is clear from the context whether we are in a two-valued or a three-valued world.

The semantics is rather standard given Table 1 due to space limitations we dispense with the formalisation. There are, however, one slightly non-standard aspect, namely the meaning of equality (denoted by the symbol '='). This comes from our desire to compactly represent multiple concrete elements with the same "abstract element". Therefore, the meaning of the predicate $=$ is defined in terms of the unary summary predicate, $s m$, that expresses that an individual represents more than one entity, and the equality, =, upon individuals:

- Non-identical individuals are not equal: $u_{1}=u_{2}$ yields 0 if $u_{1} \neq u_{2}$.

- A non-summary individual is equal to itself: $u=u$ yields 1 if $\operatorname{sm}(u)=0$.

- A summary individual may be equal to itself: $u=u$ yields $1 / 2$ if $\operatorname{sm}(u)=1 / 2$.

Here we exploit the fact that $\operatorname{sm}(u)$ is never allowed to give 1 . This will be made more precise when defining the notion of plain (two-valued) and blurred (three-valued) structures in Section 4.

Since we are interested in program analysis it is important to observe that there is an information ordering $\sqsubseteq$ where $l_{1} \sqsubseteq l_{2}$ denotes that $l_{1}$ has more definite 
Table 2. The intended meaning of the predicate symbols.

\begin{tabular}{ll} 
predicate & intended meaning \\
\hline$p a\left(v_{1}, v_{2}\right)$ & is $v_{2}$ an immediate parent of $v_{1} ?$ \\
$m(v)$ & does $v$ denote an occurrence of an ambient named $m ?$ \\
in $m(v)$ & does $v$ denote an occurrence of an action in $m ?$ \\
out $m(v)$ & does $v$ denote an occurrence of an action out $m ?$ \\
open $m(v)$ & does $v$ denote an occurrence of an action open $m ?$ \\
$!(v)$ & does $v$ denote an occurrence of a replicator operation !? \\
$\operatorname{sm}(v)$ & does $v$ represent more than one element?
\end{tabular}

information than $l_{2}$; formally, $l_{1} \sqsubseteq l_{2}$ if and only if $l_{1}=l_{2}$ or $l_{2}=1 / 2$. We write $\sqcup$ for the join operation with respect to $\sqsubseteq$. Viewing 0 as meaning $\{$ false $\}$ etc. the information ordering coincides with the subset ordering and $\sqcup$ with set-union. It is important to point out that Kleene's logic is monotonic in this order.

\section{The Abstract Domain}

Motivation. The two ambients $n[m[0]]$ and $n[m[0] \| m[0]]$ are distinct because the former has only one occurrence of $m$ inside $n$ whereas the latter has two. In other words, the collection of constituents of an ambient denote a multiset rather than a set.

To facilitate the use of classical notions from logic we want to view the collection of constituents as a set rather than as a multiset. We do this by introducing a set of individuals, so that the collection of constituents simply are a set of individuals. Informally, the above ambients will be represented as $\left(u_{1}: n\right)\left[\left(u_{2}: m\right)[0]\right]$ and $\left(u_{1}: n\right)\left[\left(u_{2}: m\right)[0] \rrbracket\left(u_{3}: m\right)[0]\right]$, respectively.

Once we have introduced the notion of individuals we are ready to model ambients by structures of the kind already mentioned in Section 3 and defined formally below. These structures are obtained by fixing the set of predicate symbols so as to be able to represent ambients; we shall use the predicates shown in Table 2. In particular, there is a binary relation $p a$ to represent the parent relation between individuals, and a number of unary relation symbols to represent the ambient information associated with individuals. Returning to the two ambients above we have that

$$
n[m[0] \rrbracket m[0]] \text { yields } \iota(p a)\left(u, u^{\prime}\right)= \begin{cases}1 & \text { if } u \in\left\{u_{2}, u_{3}\right\} \wedge u^{\prime}=u_{1} \\ 0 & \text { otherwise }\end{cases}
$$

and similarly for $n[m[0]]$.

Plain and blurred structures. We shall first fix the set of predicates to be used in the logic. Let $N$ be a finite and non-empty set of ambient names and let $\operatorname{Pr}=\operatorname{Pr}[1] \cup \operatorname{Pr}[2]$ be given by the following sets of predicates:

$$
\begin{aligned}
& \operatorname{Pr}[1]=\{s m\} \cup\{!\} \cup\{\text { in } m, \text { out } m, \text { open } m \mid m \in N\} \cup\{m \mid m \in N\} \\
& \operatorname{Pr}[2]=\{p a\}
\end{aligned}
$$




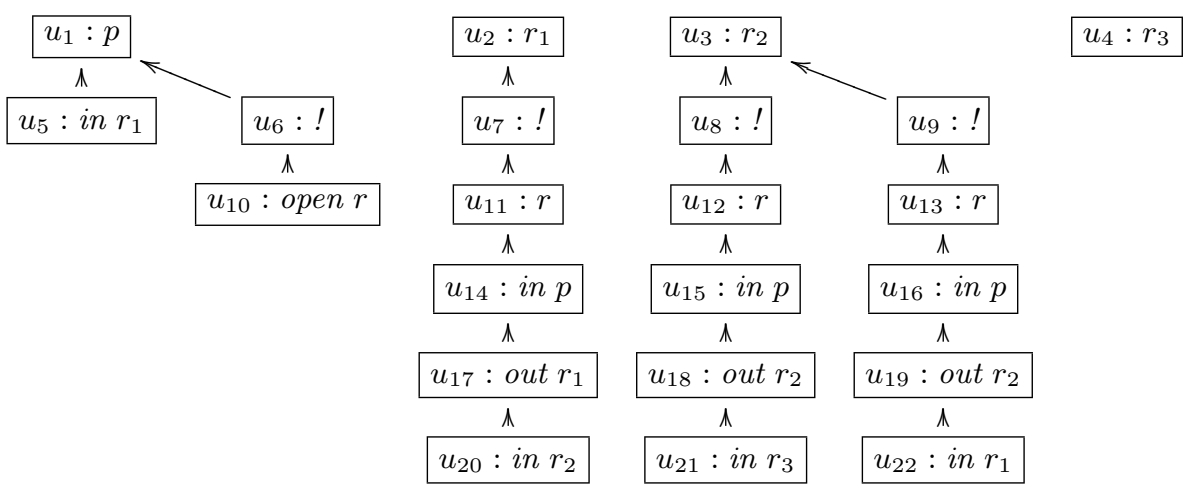

Fig. 2. A plain (two-valued) structure for the running example.

A blurred structure is then a three-valued interpretation $T=\langle U, \iota\rangle_{3}$ in the sense of Section 3 that satisfies the following conditions:

- The set $U$ is countably infinite and $\forall u \in U: \iota(s m)(u) \neq 1$.

- The set $U_{a c}$ defined below is finite:

$$
\begin{aligned}
U_{a c}=\{u \in U \mid & \exists p \in \operatorname{Pr}[1] \backslash\{s m\}: \iota(p)(u) \neq 0 \vee \\
& \left.\exists u^{\prime} \in U:\left(\iota(p a)\left(u, u^{\prime}\right) \neq 0 \vee \iota(p a)\left(u^{\prime}, u\right) \neq 0\right)\right\}
\end{aligned}
$$

A plain structure $S=\langle U, \iota\rangle_{2}$ is a two-level structure satisfying the above conditions; hence the unary predicate $s m$ maps all individuals to 0. Plain structures suffice for representing ambients precisely. Blurred structures are only needed in order to obtain a computable analysis.

Example 2. Fig. 2 shows the plain structure that corresponds to the program of Example 1. Directed edges represent the parent relation and individuals are annotated with those unary predicates that give the value 1 .

Fig. 3 shows a blurred structure for the same program. It is obtained by merging individuals from Fig. 2 that satisfy the same unary predicates. As an example the individuals $u_{5}$ ad $u_{22}$ in Fig. 2 are merged into a summary individual $u_{5,22}$ in Fig. 3, this individual is now surrounded by a dotted box since its $\mathrm{sm}$ value is $1 / 2$. Also, it has two outgoing dotted edges which describe the two potential places in the ambient hierarchy were the capability can occur; dotted edges means that the $p a$ predicate evaluates to $1 / 2$.

Representations of ambients. Table 3 defines a one-to-one (but not necessarily onto) mapping $\hat{\cdot}$ from mobile ambients into plain structures. It makes use of the operation empty that returns a structure $\langle U, \iota\rangle$ where all predicates are interpreted so that they yield 0 . The operation $n e w(p,\langle U, \iota\rangle)($ for $p \in \operatorname{Pr}[1])$ returns a structure $\left\langle U^{\prime}, \iota^{\prime}\right\rangle$ that is as $\langle U, \iota\rangle$ except that now $U_{a c}^{\prime}$ contains an 


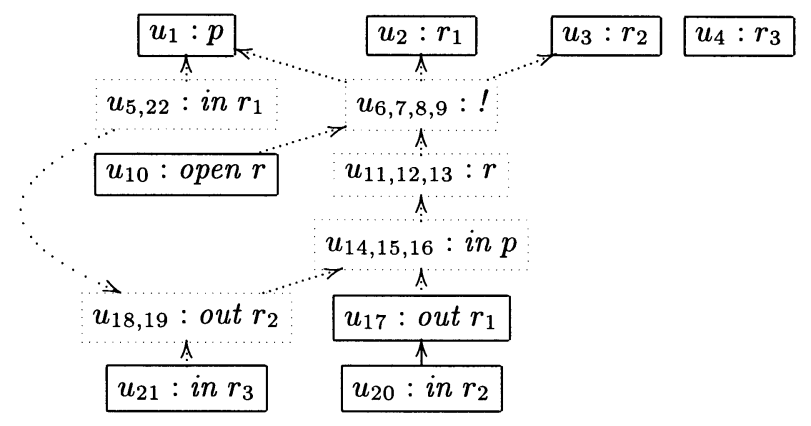

Fig. 3. A blurred (three-valued) structure for the running example.

Table 3. The mapping $\widehat{\cdot}$ from ambients to structures.

$$
\begin{aligned}
& \widehat{0}=\text { empty } \quad \widehat{\text { in } m \cdot P}=\operatorname{new}(\text { in } m, \widehat{P}) \\
& \widehat{\mathrm{m}[P]}=\operatorname{new}(m, \widehat{P}) \quad \widehat{\text { out } m . P}=\operatorname{new}(\text { out } m, \widehat{P}) \\
& \widehat{P_{1} \llbracket P_{2}}=\widehat{P_{1}} \uplus \widehat{P_{2}} \quad \text { open } m \cdot P=\text { new }(\text { open } m, \widehat{P}) \\
& \widehat{! P}=\operatorname{new}(!, \widehat{P})
\end{aligned}
$$

additional element $r$ not in $U_{a c}$; the predicate $p$ is set to 1 on $r$ and all other predicates involving $r$ are set to 0 . Some of the individuals $r^{\prime}$ of $U$ will serve as "roots" (of subprocesses represented by the structure) and the predicate $p a$ is set to 1 on the pairs $\left(r^{\prime}, r\right)$ and 0 elsewhere. We shall not formalise the concept of "roots" but only mention that the individual $r$ will be the "root" of the new structure. Finally, the operation $\langle U, \iota\rangle \uplus\left\langle U^{\prime}, \iota^{\prime}\right\rangle$ returns a structure $\left\langle U^{\prime \prime}, \iota^{\prime \prime}\right\rangle$ where $U^{\prime \prime}=U \cup U^{\prime}$ and we take case to rename the individuals of $U$ and $U^{\prime}$ such that $U \cap U^{\prime}=\emptyset$; the "roots" are the union of those of the two structures. Fig. 2 shows the result of applying $\widehat{\cdot}$ to the program of Example 1

Embeddings. Next, we define an embedding order on structures.

Definition 1. Let $T=\langle U, \iota\rangle_{\kappa}$ and $T^{\prime}=\left\langle U^{\prime}, \iota^{\prime}\right\rangle_{3}$ be two structures (for $\kappa$ being 2 or 3) and let $f: U \rightarrow U^{\prime}$ be a surjective function. We say that $f$ embeds $T$ in $T^{\prime}\left(\right.$ written $\left.T \sqsubseteq^{f} T^{\prime}\right)$ if

- for every $p \in \operatorname{Pr}[k] \backslash\{s m\}$ :

$$
\iota^{\prime}(p)\left(u_{1}^{\prime}, \ldots, u_{k}^{\prime}\right) \sqsupseteq \bigsqcup_{f\left(u_{i}\right)=u_{i}^{\prime}, 1 \leq i \leq k} \iota(p)\left(u_{1}, \ldots, u_{k}\right)
$$

- for every $u^{\prime} \in U^{\prime}$ :

$$
\iota^{\prime}(s m)\left(u^{\prime}\right) \sqsupseteq\left(\left|\left\{u \mid f(u)=u^{\prime}\right\}\right|>1\right) \sqcup \bigsqcup_{f(u)=u^{\prime}} \iota(s m)(u)
$$


The embedding is tight if equalities hold in (1) and (2).

We say that $T$ can be embedded in $T^{\prime}$ (denoted by $T \sqsubseteq T^{\prime}$ ) if there exists a function $f$ such that $T \sqsubseteq^{f} T^{\prime}$.

We can now establish an Embedding Theorem [18 that intuitively says:

If $T$ can be embedded in $T^{\prime}$, then every piece of information extracted from $T^{\prime}$ via a formula $\varphi$ is a conservative approximation of the information extracted from $T$ via $\varphi$.

The first usage of the embedding order is to define the concretization: the set of plain structures (and hence ambients) described by a blurred structure.

Definition 2. For a blurred structure $T$, we denote by $\mathcal{E}(T)$ the set of plain structures $S$ that can be embedded into $T$.

If a formula $\varphi$ evaluates to 1 over a blurred structure $T$ then it is true in all plain structures $S \in \mathcal{E}(T)$; if it evaluates to 0 then it is false in all plain structures $S \in \mathcal{E}(T)$; finally, if it evaluates to $1 / 2$ it may be true in some $S_{1} \in \mathcal{E}(T)$ and false in some $S_{0} \in \mathcal{E}(T)$.

Example 3. For the program of Example1 1 we may be interested in the properties

$$
\begin{gathered}
\text { unique } \equiv \forall v_{1}, v_{2}: p\left(v_{1}\right) \wedge p\left(v_{2}\right) \Longrightarrow v_{1}=v_{2} \\
\text { position } \equiv \forall v_{1}, v_{2}, v_{3}: p\left(v_{1}\right) \wedge p a^{+}\left(v_{1}, v_{2}\right) \wedge p a^{+}\left(v_{1}, v_{3}\right) \wedge \operatorname{ro}\left(v_{2}\right) \wedge \operatorname{ro}\left(v_{3}\right) \\
\Longrightarrow v_{2}=v_{3}
\end{gathered}
$$

where $r o(v) \equiv r_{1}(v) \vee r_{2}(v) \vee r_{3}(v)$. The formula (3) expresses that the structure only contains a single copy of the ambient $p$. The formula (41) expresses that the ambient $p$ will be within at most one of the ambients $r_{1}, r_{2}$ and $r_{3}$. These formulae have the value 1 when evaluated on the structures of Figures 2 and 3 .

Bounded Structures and Canonical Embeddings. A simple way to guarantee the termination of our analysis is by ensuring that the number of blurred structures is a priori bounded. A blurred structure $T=\langle U, \iota\rangle$ is bounded if for every two different elements $u_{1}, u_{2} \in U_{a c}$ there exists a unary predicate $p \in \operatorname{Pr}[1] \backslash\{s m\}$, such that $\iota(p)\left(u_{1}\right) \neq \iota(p)\left(u_{2}\right)$. Clearly, the number of different individuals in $U_{a c}$ is then bounded by $3^{|\operatorname{Pr}[1]|-1}=O\left(3^{|N|}\right)$ and thus the number of bounded structures is finite (up to isomorphism).

Moreover, every blurred structure can be embedded into a bounded structure by "joining" the truth-values of individuals mapping into the same abstract individual. More precisely, a special kind of tight embedding, called canonical embedding, from structures into bounded structures is obtained by defining the embedding $f$ to map individuals $u_{1}$ and $u_{2}$ in $U_{a c}$ to the same individual if and only if it is not the case that there is a predicate $p \in \operatorname{Pr}[1] \backslash\{s m\}$ such that $\iota(p)\left(u_{1}\right) \neq \iota(p)\left(u_{2}\right)$. Since the canonical embedding $f$ is uniquely defined on $T$ (up to isomorphism) we denote $f(T)$ simply as $\lfloor T\rfloor$.

Example 4. The blurred structure in Fig. 3 is the canonical embedding of the plain structure of Fig. 2. 
Table 4. Shorthand formulae used in the transition formulae.

\begin{tabular}{|c|c|c|}
\hline formula & intended-meaning & formal-meaning \\
\hline$n b(v)$ & $v$ is a non-blocking action & $\bigvee_{m \in N} m(v)$ \\
\hline$n b a(v)$ & all ancestors of $v$ are non-blocking & $\forall v_{1}: p a^{+}\left(v, v_{1}\right) \Rightarrow n b\left(v_{1}\right)$ \\
\hline $\operatorname{sib}\left(v_{1}, v_{2}\right)$ & $v_{1}$ and $v_{2}$ are sibling individuals & $\begin{array}{l}v_{1} \neq v_{2} \wedge \\
\left(\left(\exists v_{p}: p a\left(v_{1}, v_{p}\right) \wedge p a\left(v_{2}, v_{p}\right)\right)\right. \\
\left.\quad \vee \neg\left(\exists v_{p}: p a\left(v_{1}, v_{p}\right) \vee p a\left(v_{2}, v_{p}\right)\right)\right)\end{array}$ \\
\hline$a c(v)$ & $v$ is an active individual & $\begin{array}{l}\left(\bigvee_{p \in P r[1] \backslash\{s m\}} a c(v)\right) \\
\vee \exists v^{\prime}:\left(p a\left(v, v^{\prime}\right) \vee p a\left(v^{\prime}, v\right)\right)\end{array}$ \\
\hline
\end{tabular}

\section{A Simple Analysis}

We now define the effect of ambient actions by formulae that compute new structures from old. These formulae are quite natural since when interpreted over plain structures they define a semantics which is equivalent to the one of Section 2 but when interpreted over blurred structures, they are conservative due to the Embedding Theorem. Restricting our attention to bounded structures thus gives us a conservative and efficient analysis of ambients.

Capability actions. Table 5 defines the effect of capability actions using the shorthand formulae defined in Table 4. The semantics of Table 5 is formally defined in Definition 3. Informally, an action is characterized by the following kinds of information:

- The condition in which the action is enabled. It is specified as a formula with free logical variables $f_{c}, f_{1}, f_{2}, \ldots, f_{n}$ ranging over individuals. The formal variable $f_{c}$ denotes the current individual and the rest of $f_{1}, f_{2}, \ldots, f_{n}$ denote surrounding individuals. Whenever an action is enabled, it binds the free logical variables to actual individuals in the structure. Our operational semantics is non-deterministic in the sense that many actions can be enabled simultaneously and one of them is chosen for execution.

- Enabled actions create a new structure where the interpretations of every predicate $p \in \operatorname{Pr}[k]$ is determined by evaluating a formula $\varphi_{p}\left(v_{1}, v_{2}, \ldots, v_{k}\right)$ which may use $v_{1}, v_{2}, \ldots, v_{k}$ and $f_{c}, f_{1}, f_{2}, \ldots, f_{n}$ as well as all $p \in \operatorname{Pr}$.

For simplicity, our semantics does not deal with the removal of individuals (and hence our structures use an infinite set of individuals).

Consider the treatment of the in $m$ action in Table 5. It is enabled when an individual $f_{c}$ is non-blocking (i.e. when there are no capabilities or replication operators on a path to a root of the structure), it denotes an in $m$ action, it has a parent $f_{p}$, with a sibling individual $f_{s}$ which denotes an ambient named $m$. Next the enabled in $m$ action creates a parent relation, where $f_{p}$ is connected to $f_{s}$, predecessors of $f_{c}$ are connected to $f_{p}$, and individuals which are not emanating 
Table 5. The structure update formulae for executing capability actions.

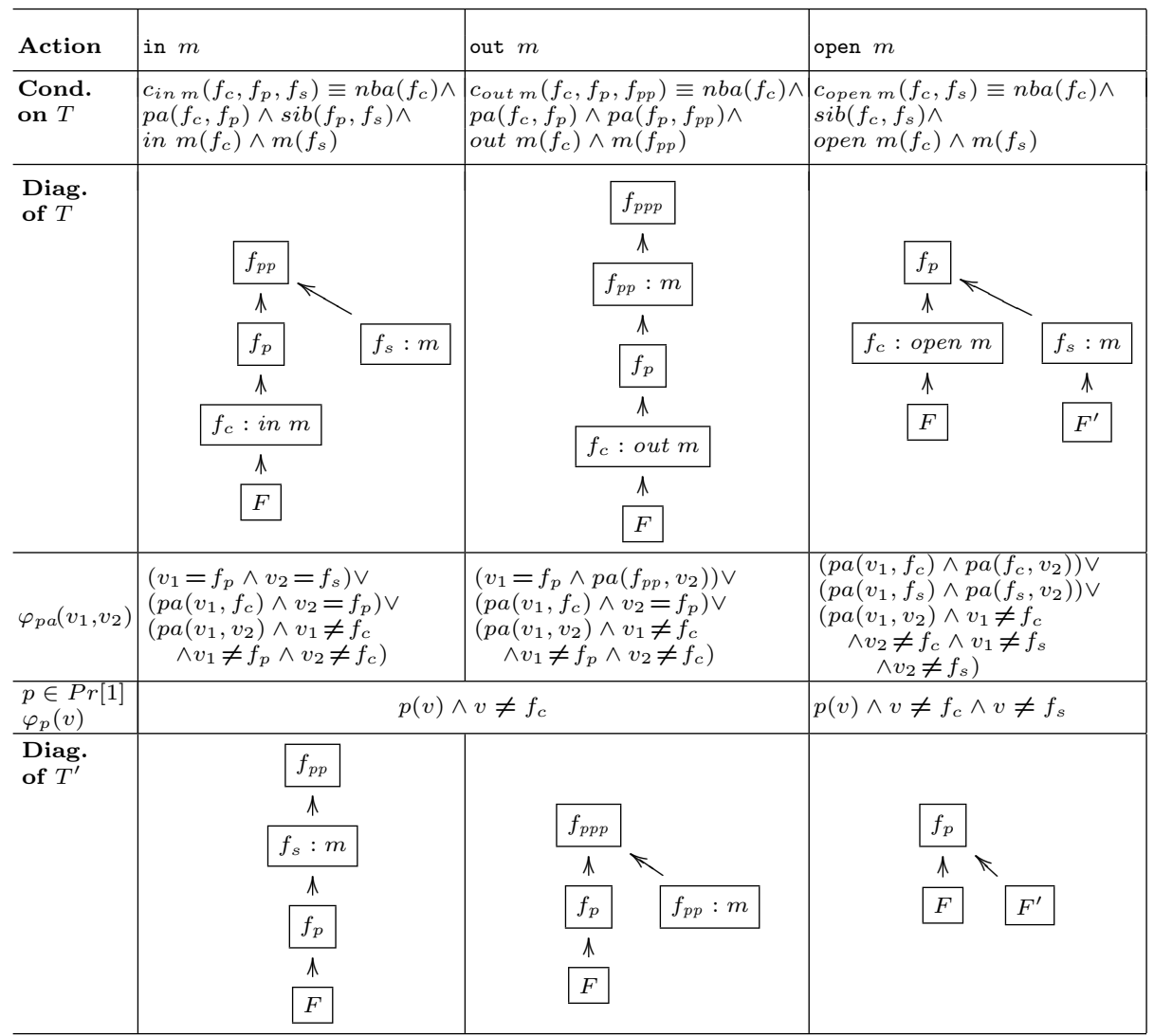

from $f_{c}$ or $f_{p}$ and not entering $f_{c}$ are not changed. On plain structures this operation exactly emulates the operational semantics (defined informally in Fig. 1). However, on blurred structure it can yield indefinite values as demonstrated in the blurred structure in Table 4, which is obtained by performing the action in $r_{1}$ on the blurred structure shown in Fig. 3

Formally, the meaning of capability actions is defined as follows:

Definition 3. We say that a $\kappa$-valued structure $T=\langle U, \iota\rangle_{\kappa}$ rewrites into a structure $T^{\prime}=\left\langle U^{\prime}, \iota^{\prime}\right\rangle_{\kappa}$ (denoted by $T \stackrel{\kappa}{\rightarrow}_{M} T^{\prime}$ ) where $M \in\{$ in $m$, out $m$, open $m \mid$ $m \in N\}$ if there exists an assignment $Z$ such that $\llbracket c_{M}\left(f_{c}, f_{1}, f_{2}, \ldots, f_{n}\right) \rrbracket_{\kappa}^{T}(Z) \neq$ 0 where the formula $c_{M}\left(f_{c}, f_{1}, f_{2}, \ldots, f_{n}\right)$ is defined in the first row of Table 5 . and for every $p \in \operatorname{Pr}[k]$ and $u_{1}, \ldots, u_{k} \in U^{\prime}$,

$$
\iota^{\prime}(p)\left(u_{1}, \ldots, u_{k}\right)=\llbracket \varphi_{p}\left(v_{1}, v_{2}, \ldots, v_{k}\right) \rrbracket_{\kappa}^{T}\left(Z\left[v_{1} \mapsto u_{1}, v_{2} \mapsto u_{2}, \ldots, v_{k} \mapsto u_{k}\right]\right)
$$

where $\varphi_{p}\left(v_{1}, \cdots, v_{k}\right)$ is the formula for $p$ given in Table 5 . 
Table 6. The structure update formulae for executing the replication operation.

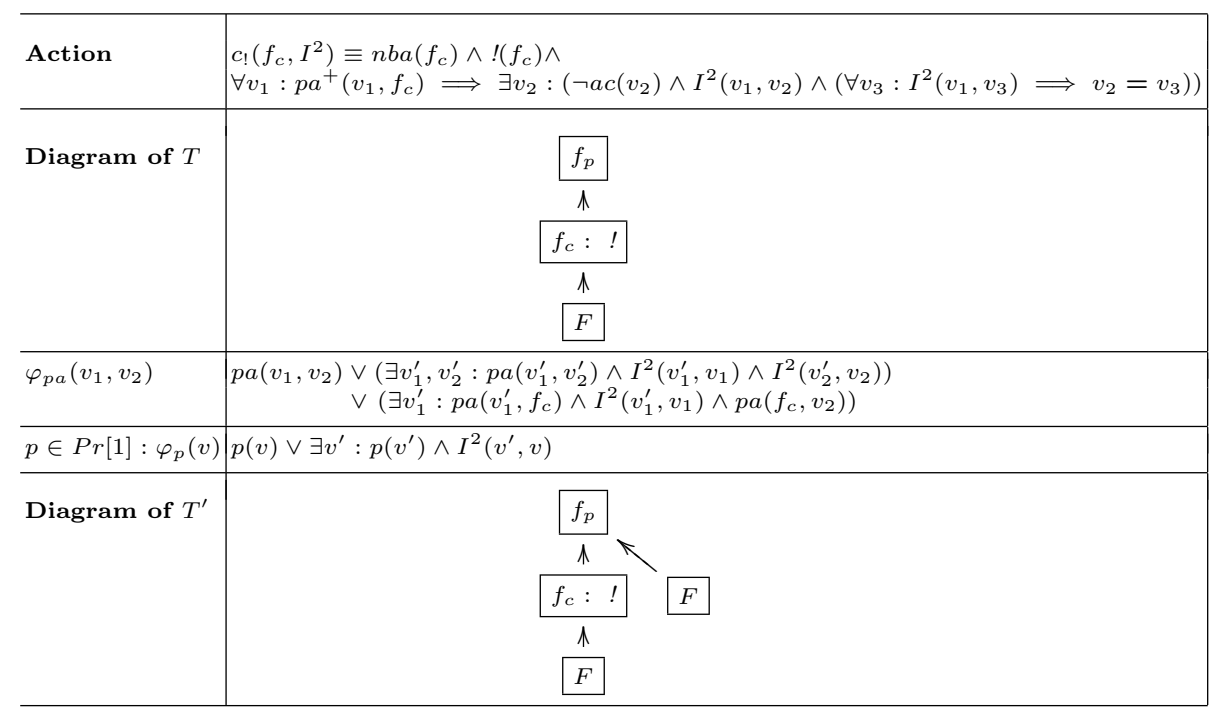

After reduction of in $r_{1}$ at $f_{c}=u_{5,22}, f_{p}=u_{1}, f_{s}=u_{2}$ :

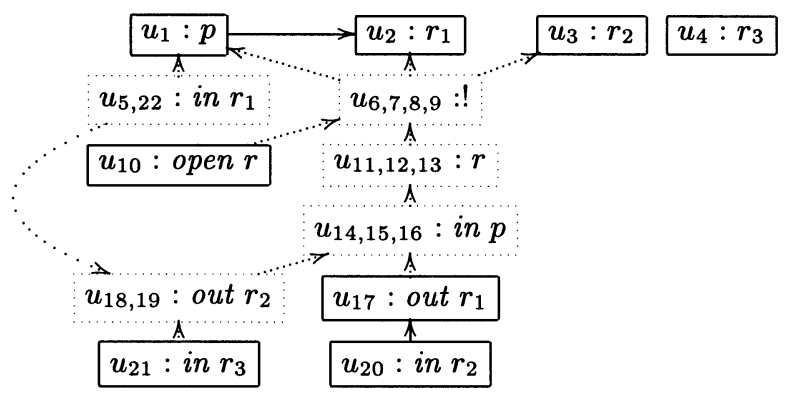

Fig. 4. The bounded structures arising in analysis after the first iteration.

Replication actions. In Table 6] we define the meaning of the replication action; we express this operation in logic by using a second order relation $I^{2}$ that creates new isomorphic individuals for the replicated ambients:

- The condition under which the operation is enabled: that the current individual $f_{c}$ is a non-blocking replication operation.

- The update formula $\varphi$. Here we use an extra preset relation $I^{2}\left(v_{1}, v_{2}\right)$ which is set to true if $v_{2}$ is a new instance of an individual $v_{1}$. This relation is set before the formulae $\varphi_{p}\left(v_{1}, v_{2}, \ldots, v_{k}\right)$ is evaluated. The formulae $\varphi_{p}\left(v_{1}, v_{2}, \ldots, v_{k}\right)$ uses $v_{1}, v_{2}, \ldots, v_{k}, f_{c}$, and the new relation $I^{2}$. 
On plain structures the replication operation exactly emulates the operational semantics. However, on blurred structure it can yield indefinite values and even more when the resultant structure is converted into a bounded one.

Formally, replication is handled as follows:

Definition 4. We say that a $\kappa$-valued structure $T=\langle U, \iota\rangle$ rewrites into a structure $T^{\prime}=\left\langle U^{\prime}, \iota^{\prime}\right\rangle \quad$ (denoted by $T \stackrel{\kappa}{\rightarrow} ! T^{\prime}$ ) if there exists an assignment $Z$ such that $\llbracket c_{!}\left(f_{c}, I^{2}\right) \rrbracket_{\kappa}^{T}(Z) \neq 0$ where the formula $c_{!}\left(f_{c}, I^{2}\right)$ is defined in the first row of Table [6, and for every $p \in \operatorname{Pr}[k]$ and $u_{1}, u_{2}, \ldots, u_{k} \in U^{\prime}$,

$\iota^{\prime}(p)\left(u_{1}, u_{2}, \ldots, u_{k}\right)=\llbracket \varphi_{p}\left(v_{1}, v_{2}, \ldots, v_{k}\right) \rrbracket_{\kappa}^{T}\left(Z\left[v_{1} \mapsto u_{1}, v_{2} \mapsto u_{2}, \ldots, v_{k} \mapsto u_{k}\right]\right)$ where $\varphi_{p}\left(v_{1}, \cdots, v_{k}\right)$ is the formula for $p$ given in Table 6 .

Finally, we can formally define the analysis (or abstract semantics):

Definition 5. We say that a $\kappa$-valued structure $T=\langle U, \iota\rangle$ rewrites into a structure $T^{\prime}=\left\langle U, \iota^{\prime}\right\rangle \quad$ (denoted by $T \stackrel{\kappa}{\rightarrow} T^{\prime}$ ) if either $T \stackrel{\kappa}{\rightarrow}_{M} T^{\prime}$ or $T \stackrel{\kappa}{\rightarrow} T^{\prime}$. We say that $T$ canonically rewrites into $T^{\prime}$ (denoted by $T \stackrel{\lfloor 3\rfloor}{\rightarrow} T^{\prime}$ ) when $T=\lfloor T\rfloor$ and there exists $T^{\prime \prime}$ such that $T^{\prime}=\left\lfloor T^{\prime \prime}\right\rfloor$ and $T \stackrel{3}{\rightarrow} T^{\prime \prime}$. We denote by $\stackrel{3}{\rightarrow}^{*}$ the reflexive transitive closure of $\stackrel{3}{\rightarrow}$ and similarly for $\stackrel{2}{\rightarrow}$ and $\stackrel{\lfloor\rfloor^{*} *}{\rightarrow}$.

Properties of the Abstract Semantics. The set of bounded structures

$$
\left.A n_{T} \stackrel{\left\lfloor 3 \downarrow^{*}\right.}{\rightarrow} T^{\prime}\right\}
$$

is finite and can be computed iteratively in a straightforward manner.

We can show that the semantics of processes, $\widehat{P} \rightarrow{ }^{*} \widehat{Q}$, is correctly modelled by our plain rewrite relation, $\widehat{P} \stackrel{2}{\rightarrow}^{*} \widehat{Q}$. It then follows from the Embedding Theorem that the semantics of processes is correctly modelled by our blurred rewrite relation:

$$
\text { Whenever } P \rightarrow{ }^{*} Q \text { we have } \exists T \in A n_{\lfloor\widehat{P}\rfloor}: \widehat{Q} \sqsubseteq T \text {. }
$$

Thus, we can verify safety properties of ambients by evaluating formulae against blurred structures in $A n_{T}$. Of course, $A n_{T}$ may also include superfluous structures leading to imprecise results.

\section{Conclusion}

So far we have presented a very naive analysis of ambients (in the spirit of the shape analysis algorithm in [5. Section 3]). The motivation was to show the benefit of three-valued logics. We have implemented the analysis using the TVLA system [12] but in its current form it is too costly and too imprecise to give informative answers to the questions asked of the running example.

To get an efficient and sufficiently precise analysis we have refined the analysis by using two techniques already mentioned in [18]. One is to maintain finer distinctions on blurred structures based on values of so-called instrumentation predicates and the other is to control the complexity by joining structures. 
Table 7. Definition of instrumentation predicates.

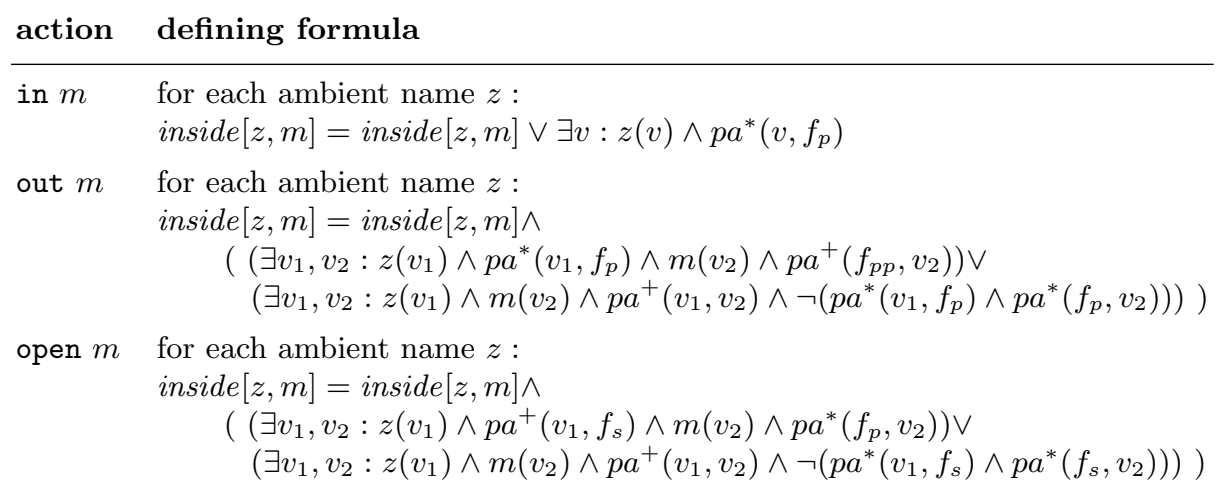

Instrumentation predicates. Technically, instrumentation predicates are just predicates which store some context information. For our analysis we have added two kinds of instrumentation predicates. One group of predicates simply labels the individual ambients and capabilities of the program much as in 916. As an example, the initial blurred structure will now be similar to the initial plain structure displayed in Fig. 2 as now the active individuals will have distinct labels and hence will not be combined. The labels will remain unchanged throughout the analysis and when a part of a structure is copied as in the analysis of replication the new individuals inherit the labels of the original individuals. The benefit of adding these instrumentation predicates is that the analysis can better distinguish between the different routers in which the packet resides.

Another group of instrumentation predicates are designed to reflect the three questions we have asked which all are concerned about which ambients are inside which ambients. We therefore define nullary predicatesinside $\left[z_{1}, z_{2}\right]$ for each combination of the ambient names $\left(z_{1}, z_{2}\right) \in N \times N$; it is defined by

$$
\text { inside }\left[z_{1}, z_{2}\right]=\exists v_{1}, v_{2}: z_{1}\left(v_{1}\right) \wedge z_{2}\left(v_{2}\right) \wedge p a^{+}\left(v_{1}, v_{2}\right)
$$

and it is updated whenever one of the capabilities are executed as shown in Table [7] it is unchanged when the replication action is executed.

Joining structures. While the goal of adding instrumentation predicates is to get more precision, the goal of joining structures is to get more efficiency and as usual this means that we are going to loose precision. We therefore merge structures satisfying the same nullary instrumentation predicates.

With these modifications the system of [12] can indeed validate the two properties of Example 3. This took 192.6 CPU seconds on a Pentium 256 Mhz machine running NT 4.0 with JDK 1.2.

Acknowledgements. The running example was suggested by Luca Cardelli. Tal Lev-Ami provided the implementation discussed in Section 6. 


\section{References}

1. L. Cardelli, A. D. Gordon: Mobile ambients. In Proc. FoSSaCS'98, vol. 1378 of LNCS, pages 140-155, Springer, 1998.

2. L. Cardelli, A. D. Gordon: Types for mobile ambients. In Proc. POPL'99, ACM Press, 1999.

3. L. Cardelli, G. Ghelli, A. D. Gordon: Mobility types for mobile ambients. In Proc. ICALP'99, LNCS, Springer, 1999.

4. L. Cardelli and A. D. Gordon: Anytime, Anywhere: Modal Logics for Mobile Ambients. In Proc. POPL'00, ACM Press, 2000.

5. D.R. Chase, M. Wegman, and F. Zadeck. Analysis of pointers and structures. In SIGPLAN Conf. on Prog. Lang. Design and Impl., pages 296-310, ACM Press, 1990.

6. P. Cousot and R. Cousot. Systematic design of program analysis frameworks. In Symp. on Princ. of Prog. Lang., pages 269-282, ACM Press, 1979.

7. R.L. Epstein. The Semantic Foundations of Logic, Volume 1: Propositional Logics. Kluwer Academic Publishers, 1990.

8. M.L. Ginsberg. Multivalued logics: A uniform approach to inference in artificial intelligence. In Comp. Intell., 4:265-316, 1988.

9. R. R. Hansen, J. G. Jensen, F. Nielson, H. Riis Nielson: Abstract Interpretation of Mobile Ambients. In Proc. SAS'99, vol. 1694 of LNCS, Springer, 1999.

10. H. Hoddes. Three-Valued Logics: An Introduction, A Comparison of Various Logical Lexica, and Some Philosophical Remarks. In Annals of Pure and Applied Logic, 1989.

11. S.C. Kleene. Introduction to Metamathematics. North-Holland, second edition, 1987.

12. T. Lev-Ami: TVLA: a framework for Kleene based static analysis. M.Sc. thesis, Tel Aviv University, January 2000.

13. F. Levi and D. Sangiorgi: Controlling Interference in Ambients. In Proc. POPL'00, ACM Press, 2000.

14. F. Nielson: Semantics-Directed Program Analysis: A Tool-Maker's Perspective. In Proc. SAS'96, vol. 1145 of LNCS, Springer, 1996.

15. F. Nielson, H. Riis Nielson, C. L. Hankin: Principles of Program Analysis, Springer, 1999.

16. F. Nielson, H. Riis Nielson, R. R. Hansen, J. G. Jensen: Validating Firewalls in Mobile Ambients. In Proc. CONCUR'99, vol. 1664 of LNCS, Springer, 1999.

17. F. Nielson, H. Riis Nielson: Shape Analysis for Mobile Ambients. In Proc. POPL'00, ACM Press, 2000.

18. M. Sagiv, T. Reps, R. Wilhelm: Parametric Shape Analysis via 3-Valued Logic. In Proc. POPL'99, ACM Press, 1999. 